\title{
Chemical tradeoffs in seed dispersal: defensive metabolites in fruits deter consumption by mutualist bats
}

\author{
Susan R. Whitehead, Maria F. Obando Quesada and M. Deane Bowers \\ S. R. Whitehead (susan.whitehead@colorado.edu) and M. D. Bowers, Ecology and Evolutionary Biology, Univ. of Colorado, UCB 334, Boulder, \\ CO 80309, USA. MDB also at: Museum of Natural History, Univ. of Colorado, UCB 218, Boulder, CO 80309, USA. - M. F. O. Quesada, \\ Escuela de Ciencias Biológicas, Univ. Nacional de Costa Rica, Heredia, Costa Rica.
}

\begin{abstract}
Although fleshy fruits function primarily to attract seed dispersers, many animal-dispersed fruits contain potentially toxic secondary metabolites. These metabolites can provide defense against seed predators and pathogens, but their effects on dispersers are still poorly understood. In some cases plants may experience a tradeoff, where the metabolites that provide fruit defense also reduce seed disperser preferences. In other cases the bioactivity of fruit secondary metabolites may be directed primarily at pests with no negative effects on seed-dispersing vertebrates. We tested the effects of amides, a group of nitrogen-based defensive compounds common in the plant genus Piper (Piperaceae), in interactions with the primary seed dispersers of Piper in the neotropics - fruit-feeding bats in the genus Carollia (Phyllostomidae). In a series of flight cage experiments, pure amides and amide-rich fruit extracts reduced the preferences of bats for Piper fruit, affecting both the bats' initial choices to remove Piper infructescences and the proportion of fruit consumed from individual infructescences once they were removed. However, the effects of amides varied considerably among three species of Carollia and among the specific individual amides and extracts tested. Overall, our results support the hypothesis that plants experience a tradeoff between seed dispersal and fruit defense, but the strength of this tradeoff and the overall fitness consequences may depend strongly on ecological context.
\end{abstract}

The primary function of ripe, fleshy fruits is to attract mutualistic animal consumers, who contribute to plant reproductive success by dispersing seeds to new sites (van der Pijl 1982). The evolutionary history between plants and mutualist seed dispersers has led to suites of fruit traits that include both attractants (e.g. colors, odors) and nutritional rewards (e.g. proteins, lipids, sugars). However, many ripe, fleshy fruits also contain potentially deterrent or toxic secondary metabolites (Herrera 1982, Levey et al. 2007), in some cases at concentrations and levels of chemical diversity that exceed those in leaves and other plant parts (Whitehead and Bowers 2013, Whitehead et al. 2013). The occurrence of toxic secondary metabolites in a tissue meant to attract mutualists is puzzling, but a number of different adaptive hypotheses have been proposed to explain how these compounds may increase plant fitness (Cipollini and Levey 1997a). Perhaps the most well-supported of these is the defense tradeoff hypothesis, which suggests that fruit secondary metabolites function primarily to defend against non-dispersing insect and microbial fruit pests (Herrera 1982, Cipollini and Levey 1997a, Izhaki 2002, Tewksbury et al. 2008, Whitehead and Bowers 2013, 2014). However, because the same secondary metabolites that defend against pests could also affect interactions with mutualists, understanding the overall ecological and evolutionary consequences of fruit chemical defense requires a broad view of the possible effects of defense traits on the foraging and feeding behavior of seed-dispersing animals.

There are two basic hypotheses for how defensive secondary metabolites that occur in fruits could affect the triad of interactions among plants, mutualist seed dispersers, and antagonistic fruit pests. One possibility is that plants may experience an adaptive tradeoff between defense and dispersal, where secondary metabolites are ecologically costly in terms of reduced preferences of mutualist seed dispersers, but these costs are outweighed by the benefits of increased fruit defense against pests (Herrera 1982, Cipollini and Levey 1997a). Here, secondary metabolites are expected to have broad-spectrum bioactivity against fruit consumers, including fungi, bacteria, invertebrates, and vertebrates. Alternatively, plants may produce secondary metabolites in fruits that are bioactive against invertebrate and microbial antagonists, but have neutral effects in interactions with vertebrate seed dispersers. From the plant perspective, producing defensive compounds with pest-specific bioactivity would likely provide the maximum net fitness benefit, and, because seed dispersers (mostly vertebrates) are generally only distantly related to fruit pests (mostly invertebrates and microbes), it seems probable that plants could evolve suites of secondary metabolites in fruits that effectively protect 
against pests with minimal effects on dispersers (Cipollini and Levey 1997a). This scenario was originally described as the 'microbe-pest specificity model' within the defense tradeoff hypothesis framework by Cipollini and Levey (1997a). However, there is no actual expectation of a tradeoff, because there would be no ecological costs associated with reduced preferences of mutualistic seed dispersers. Thus, for the purposes of this study, we will consider the microbe-pest specificity hypothesis as a distinct alternative to the defense tradeoff hypothesis and compare support for each.

The few studies that have addressed one or both of these two alternative hypotheses have provided mixed results. Some support for the idea of a tradeoff between seed dispersal and fruit defense is provided by evidence that fruits that are high in secondary metabolites are the least preferred by seed dispersers in natural populations (Cipollini and Levey 1997b, Schaefer et al. 2003, Cazetta et al. 2008, Whitehead and Poveda 2011). However, in other cases, seed dispersers may consume fruits high in secondary metabolites with relative impunity. For example, capsaicinoids in wild chili fruits provide important defense against pathogenic fungi (Tewksbury et al. 2008, Haak et al. 2012), but do not appear to reduce consumption by seed-dispersing birds (Mason et al. 1991, Tewksbury and Nabhan 2001). However, capsaicin has been shown to deter consumption by other vertebrates, such as rodents, which also consume chili fruits but are much less efficient seed dispersers than their avian counterparts (Mason et al. 1991, Tewksbury and Nabhan 2001). There are other examples of secondary metabolites that are highly toxic to mammals but are readily consumed by birds, such as amygdalin, a cyanogenic glycoside found in fruits of the Rosaceae and Caprifoliaceae, which cedar waxwings can consume at levels equivalent to 5.5 times the oral lethal dose for rats with no outward signs of toxicity (Struempf et al. 1999). These results emphasize that the effects of fruit secondary metabolites on vertebrate preferences, and therefore the potential for tradeoffs in fruit defense, can vary considerably depending on the consumer and plant species involved (Mason et al. 1991, Cipollini and Levey 1997a, Struempf et al. 1999, Tewksbury and Nabhan 2001, Fedriani and Boulay 2006, Karasov et al. 2012).

Most past work on the ecological role of fruit secondary metabolites has focused on bird-dispersed species (Cipollini 2000, Levey et al. 2007); however, mammals also provide critical seed dispersal services for many plants (van der Pijl 1982). Particularly in tropical forests, mammals, and especially bats, are among the most abundant frugivorous animals and are critically important in forest regeneration and succession (Muscarella and Fleming 2007). Because bats forage at night, bat-dispersed fruits are expected to contain higher levels of volatile secondary metabolites that contribute to fruit odor and provide foraging cues (van der Pijl 1982, Hodgkison et al. 2007, Lomáscolo et al. 2010). However, if bats are similar to other mammals in that they are less adept at detoxifying secondary metabolites than birds (Mason et al. 1991, Cipollini and Levey 1997a, Struempf et al. 1999, Tewksbury and Nabhan 2001, Fedriani and Boulay 2006), the opposite may be true for non-volatile secondary metabolites that function primarily in fruit defense.

One important group of bat-dispersed plants is the genus Piper (Piperaceae), a diverse and dominant component of neotropical plant communities. Many Piper species fruit in abundance, producing distinctive, green, spike-shaped infructescences that are quickly removed by frugivorous bats (Fig. 1). In particular, a small genus of fruit bats (Carollia spp., Phyllostomidae) are the primary dispersers of neotropical Piper, and Piper fruits represent a year-round dietary staple for the bats, making this interaction one of the few examples of a relatively specialized seed dispersal mutualism (Fleming 2004). Past work on the phytochemistry of Piper has focused primarily on leaves (Parmar et al. 1997, Dyer et al. 2004, Kato and Furlan 2007); however, fruits of many Piper species also contain diverse mixtures of secondary metabolites, and are particularly rich in amides (Whitehead et al. 2013). Amides are a large group of nitrogen-based compounds that are known to defend Piper leaves against herbivores (Dyer et al. 2004), and also have recently been shown to function in fruit defense against insect seed-predators and fruitassociated fungi (Whitehead and Bowers 2014). The effects of amides on insects and fungi are complex, in part because amides often occur in diverse mixtures, and combinations of compounds can have non-additive effects, either functioning synergistically or antagonistically in fruit defense (Whitehead and Bowers 2014). It is unknown whether these same defensive compounds can affect Carollia bat foraging and feeding behavior.

In this study, we compared support for the defense tradeoff hypothesis versus the microbe-pest specificity hypothesis by examining the effects of two pure amides (piperine and piplartine) and amide-rich extracts from unripe and ripe fruits of Piper reticulatum on the foraging and feeding behavior of three species of Carollia bats. Piperine and piplartine were chosen for experimentation because they occur in many species of Piper, are often found in high concentrations in fruit, and are available commercially in pure form (Parmar et al. 1997, Rajopadhye et al. 2011, Bezerra et al. 2013). However, because amides in fruits can occur in complex mixtures, we also tested the effects of fruit extracts from Piper reticulatum, a common understory Piper that produces fruits containing complex mixtures of up to 30 individual amides (Whitehead et al. 2013), although it does not contain piperine or piplartine. Piper reticulatum amides are reduced by

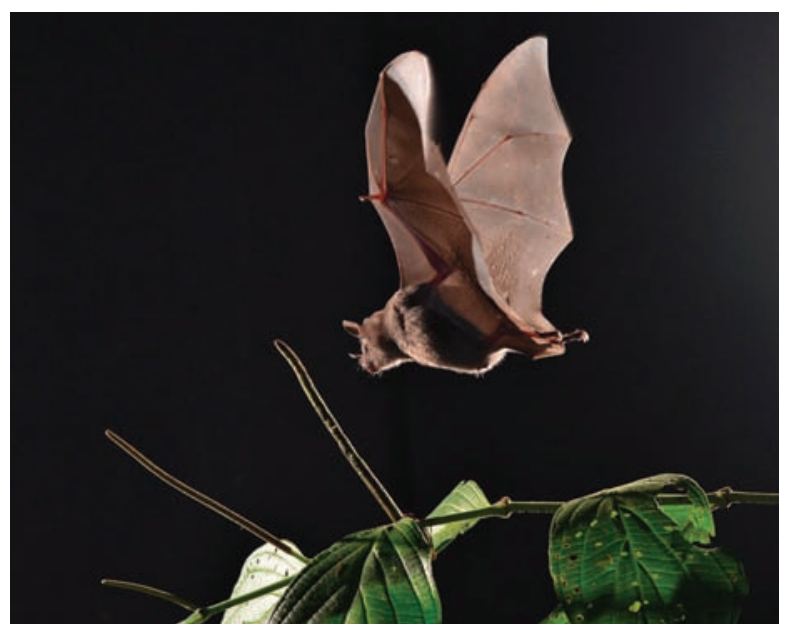

Figure 1. Carollia perspicillata removing a ripe infructescence of Piper sancti-felicis. Photo credit: S. R. Whitehead. 
nearly 50\% during fruit ripening (Whitehead et al. 2013), which may be one important reason why unripe fruits are entirely avoided by Carollia bats (Thies and Kalko 2004, Whitehead unpubl.). Our previous work has also shown that piperine, piplartine, and $P$. reticulatum extracts all have negative effects on fruit pests that are associated with Piper - the pure compounds and extracts are all strongly anti-fungal and the pure compounds reduce the feeding preferences of an insect seed predator (Whitehead and Bowers 2014). If the same compounds and extracts also reduce the preferences of seed-dispersing bats, fruit amides likely represent a tradeoff between seed dispersal and fruit defense. Alternatively, bat feeding preferences may be unaffected by fruit amides, lending support to the microbe-pest specificity hypothesis.

Carollia bats locate ripe infructescences based primarily on olfaction (Thies et al. 1998). They remove entire infructescences in flight and carry them to nearby feeding roosts for consumption. Thus, amides could affect bat preferences at two stages of feeding - infructescence removal or the consumption of fruit from an infructescence once it is removed. We examined both of these aspects of Carollia feeding behavior, as well as the potential for differences among Carollia species in their response to amides. Specifically, we asked three questions exploring the role of amides in the feeding preferences of the three species of Carollia occurring at our study site: Q1) are bats able to detect amides prior to infructescence removal and choose which infructescences to remove based on their occurrence? Q2) Do amides affect the proportion of fruit consumed from an infructescence once it is removed? Q3) Are the effects of amides on bat preferences dose-dependent within the range of variation present in natural ripe fruits?

\section{Material and methods}

\section{Study site and system}

All experiments were conducted at La Selva Biological Station, located in the Heredia province of Costa Rica, between May-June 2011 and April-July 2012. The reserve consists of 1600 hectares of protected area that includes primary tropical wet and premontane forest as well as secondary forest and abandoned agricultural areas. The site is a high center of diversity for Piper, with $50+$ species co-occurring (OTS 2012). Most Piper species at the site are dispersed primarily by bats, although a few rely almost exclusively on asexual reproduction (Greig 1993) and some are dispersed by a mix of birds and bats (Palmeirim et al. 1989). Piper fruits are borne on distinctive spike-shaped infructescences (Fig. 1) that can contain hundreds to thousands of tiny individual fruits. For most species, fruits are produced in several seasonal peaks during the year (Thies and Kalko 2004). Individual infructescences can develop slowly over a period of one month or more, after which they generally enter a rapid final ripening period where all of the fruits on an infructescence soften and swell. In many Piper species, this process begins in the early afternoon and the infructescences are generally fully ripe by dusk (Thies and Kalko 2004). The majority of infructescences are removed by bats the same evening that they ripen; those that are not removed begin to rot very quickly and are usually not removed on the following evening (Thies and Kalko 2004, Whitehead unpubl.).

Three species of Carollia bats co-occur at the site: C. perspicillata, C. sowelli and C. castanea. All three species are highly abundant in the forest understory and are among the most commonly captured bats in mist-nets. There is some evidence that the three species differ in their degree of specialization on Piper. In a previous study conducted at the same site, the percentage of Piper in the diet of these species was estimated as $\sim 54 \%$ for $C$. perspicillata, $\sim 63 \%$ for $C$. sowelli, and $\sim 85 \%$ for C. castanea (Fleming 1991).

\section{Field capture and handling of bats}

Bats were captured in mist nets from secondary forest sites at La Selva using standard methods (Kunz and Parsons 2009). Reproductive females were released and all males and nonreproductive females were retained for use in experiments. Bats were placed in cloth bags prior to the start of the experiments for a minimum of $45 \mathrm{~min}$ and a maximum of two hours. Bats were then transferred to $2.5 \times 1.5 \times 1.75 \mathrm{~m}$ tall flight cages that consisted of a wood frame with screen walls and ceilings. Each species of Carollia was housed in a separate cage. Conspecific groups were placed together, with groups ranging in size from 1-6 individuals depending on the number of bats captured in a particular evening. Each cage was equipped with a LED infrared lamp and a digital video camera to record bat behaviors (sample video in Supplementary material Appendix 1). Infrared lights and cameras are commonly used in captive studies and do not appear to disturb normal bat behavior (Kunz and Parsons 2009). Each bat was also marked individually with temporary infrared-reflective tags to allow detailed analyses of behaviors of individual bats (Supplementary material Appendix 3-4).

\section{Effects of amides on fruit removal and consumption (Q1 and Q2)}

To test whether amides affect infructescence removal (Q1) and/or consumption (Q2), we conducted a series of choice experiments testing the effects of pure amides and amiderich extracts on the preferences of three species of Carollia. In all experiments, we added pure amides or extracts to ripe fruits of P. sancti-felicis, a commonly occurring species at the study site that produces fruits in abundance continuously throughout the year. The fruits of $P$. sancti-felicis contain no detectable amides at a detection limit of approximately $0.01 \%$ dry weight, although they may contain other secondary metabolites (Whitehead 2013). Supplementing natural Piper fruits meant that we were unable to control for any variation in nutritional or secondary chemistry among $P$. sancti-felicis fruits that existed prior to our amide supplementation treatments; however, this method allowed us to observe how amides affect the natural feeding behavior of Carollia bats, including Piper infructescence removal and fruit consumption, which could not be simulated using artificial fruits that did not have the same structure as a Piper infructescence.

Five sets of identical trials were conducted to test the effects of five different amide treatments - piperine, piplartine, the 
combination of piperine and piplartine, $P$. reticulatum unripe fruit extracts and $P$. reticulatum ripe fruit extracts. Piperine and piplartine have not, to date, been identified or quantified from any of the Piper species that occur at La Selva, but they do co-occur in at least one species (Piper tuberculatum) that is common in many parts of Costa Rica. In the trials with the pure compounds, we added $10 \mathrm{mg}(\sim 2 \%$ dry weight) of piperine, piplartine, or 1:1 piperine:piplartine to each $P$. sancti-felicis infructescence. Assuming all of the added solution was absorbed by the fruit, the amount we added would mimic the concentration of total amides in $P$. tuberculatum fruits reported in other studies (1.86-2.81\% DW; Leitão da-Cunha and de Oliveira Chaves 2001, de Oliveira Chaves et al. 2003) and represents the lower end of the range of concentrations of piperine in fruits of old world Piper species (Rajopadhye et al. 2011). For the extracts, we added the amount of extract obtained from the dry weight equivalent of a single infructescence (approximately $0.5 \mathrm{~g}$ ) of P. reticulatum, which contained an estimated $8.95 \mathrm{mg}$ of total amides for unripe fruit extracts and $7.38 \mathrm{mg}$ of total amides for ripe fruit extracts. Complete procedures for amide extraction and quantification based on gas chromatography/mass spectrometry (GC/MS) are provided in the Supplementary material Appendix 2) and are similar to methods used for previous studies (Whitehead et al. 2013, Whitehead and Bowers 2014).

To prepare infructescences for the trials, we always collected freshly-ripened $P$. sancti-felicis infructescences on the afternoon before the trial began. In all treatments, the pure compounds or extracts were dissolved in ethanol and $P$. sancti-felicis infructescences were supplemented by adding $1 \mathrm{~mL}$ of solution to a clean glass petri dish and rolling a single ripe infructescence in the dish until all of the solution was absorbed and evenly coated the surface of the fruits. Control infructescences were treated in an identical manner using ethanol only. The infructescences were then left to dry on a wire rack in an air-conditioned laboratory for 3-5 h to allow the ethanol to evaporate prior to the start of the trials. Although bats can detect ethanol at low concentrations (Laska 1990), the infructescences appeared completely dry by the end of this period and we assumed that any differential evaporation between control and treatment infructescences was negligible.

To test the effects of amides on bat feeding preferences, groups of one to six conspecific bats per cage (depending on the number caught on a particular evening) were offered a buffet-style presentation of equal numbers of amidesupplemented and control infructescences. In each cage, five control infructescences were placed together in a group, and five treatment infructescences were placed together in a separate group on the opposite side of the cage. The positions of control and experimental groups were randomized nightly. Infructescences were placed in groups to simulate a foraging environment where fruits displayed together on the same Piper shrub may share particular chemical traits (e.g. high or low amide content). Trials ran for two hours, during which individual bats generally removed between one and three infructescences. In a few trials, a group of bats removed all of the infructescences from either the control or treatment group prior to the end of the two hour period, in which case we ended the trial early and excluded all removal and consumption events from analysis that occurred after one of the fruit groups was depleted. Once the experiment was complete, all intact and discarded infructescences were recovered from the cage. To estimate the proportion consumed of each infructescence, we measured the total length of the rachis and the length from which fruit had been consumed (bats leave the peduncle and central rachis intact even when the fruit is entirely consumed). The mass of fruit consumed was estimated from the length measurements using a simple linear conversion of mass $=0.0296 \times$ length -0.891 , which was determined based on an independent dataset of length and mass measured on 50 infructescences of $P$. sancti-felicis (Baldwin and Whitehead 2015a). After the experiments, bats were marked by cutting a small section of fur from the back and released at the site of capture. Marked bats that were recaptured on subsequent evenings were not used in additional trials for the same amide treatments, which were generally conducted within a period of one month. However, because tests of different compounds and extracts were conducted over 15 months across two field seasons, it is possible that some bats used in later trials were re-captures that no longer had visible markings.

In our main analyses of the effects of amides on removal and consumption (Q1 and Q2), we controlled for the potential non-independence of feeding preferences among bats within a group by pooling data by trial for all bats that were housed together. However, we also recorded data for each bat individually by marking all bats and all infructescences with unique markings (Supplementary material Appendix 3-4). This allowed us to examine whether the effects of amides varied based on individual bat traits such as sex, age, weight, and reproductive status (Supplementary material Appendix 3). In addition, we were able to analyze whether amide preferences changed over the course of the trial based on individual experience or the experiences of other bats in the same flight cage (Supplementary material Appendix 4).

\section{Dose-dependent effects of ripe fruit amides on fruit consumption $(\mathrm{Q} 3)$}

To further explore whether there were dose-dependent effects of the specific amides present in $P$. reticulatum ripe fruits (Q3), we conducted a separate no-choice feeding experiment that examined the effects of ripe fruit extracts presented in varying concentrations on fruit consumption by $C$. perspicillata. This study required bats to be housed in flight cages for multiple days, and C. perspicillata was chosen for continued study because of the relative ease with which it can be maintained in captivity using bananas as a maintenance diet between trials. For these experiments, we housed seven individuals of $C$. perspicillata in seven smaller solitary flight cages $(2 \times 1 \times 1 \mathrm{~m}$ tall $)$ over a five day period. On the evening of capture, bats were transferred to cages and given a maintenance diet of ripe bananas and water ad libitum until the evening that trials began. On each evening of the trial period, we presented the bat with five $P$. sancti-felicis infructescences that were all treated in an identical manner. On successive evenings, bats were offered control infructescences (treated with ethanol only, as above), infructescences supplemented at a concentration typical of $P$. reticulatum (1X; identical to the concentration used in the choice experiments 
described above), infructescences supplemented at one tenth that concentration $(0.1 \mathrm{X})$, and infructescences supplemented at twice that concentration $(2 \mathrm{X})$. All of the treatment concentrations $(0.1 \mathrm{X}-2 \mathrm{X})$ are within the range of variation present in natural populations of $P$. reticulatum (Whitehead et al. 2013). The order of presentation of these treatments was randomized for each individual bat. Bats were allowed to feed from the Piper infructescences for a period of two hours from the start of typical foraging (around 18:00 h), after which we returned to the cage, removed all intact and discarded infructescences, measured the total length and length consumed from each, and estimated the total mass consumed as above. The maintenance diet of ripe bananas was then offered ad libitum for the remainder of the evening. Any remaining bananas were removed the following day prior to the start of foraging to ensure that the experimental Piper fruit was the first meal of the evening for the duration of the trials. All bats were released at the site of capture following the trial period.

\section{Statistical analyses}

To test whether amides affect infructescence removal by Carollia bats (Q1), we used data that were pooled by trial and created an index of removal preference, calculated for each trial as the total number of treatment minus control infructescences removed by the entire group of bats during the trial period. This was done to avoid any statistical issues of non-independence between choices of individual bats housed together or between removal rates of two food types offered simultaneously, a common difficulty in the analysis of choice tests (Roa 1992, Larrinaga 2010). To determine whether there was an overall effect of amides on removal, we used a one-sample t-test that compared the distribution of the removal preference index to zero. This initial analysis was conducted with data from all trials, combined without respect to amide treatment or bat species (see Supplementary material Appendix 5 for preference indices calculated separately for each combination of amide treatment and bat species). We then used a general linear model (GLM) to compare among the different amide treatments and determine whether amide treatment, bat species, group size, and their interactions affected the magnitude of the removal preference index. We began with a saturated model that included all two and three-way interactions and then used a model simplification approach (Crawley 2007) to determine the minimum adequate model (Table 1). After finding a significant interaction between amide treatment and bat species, we analyzed differences among bat species in their removal preferences for each amide treatment separately. For each amide treatment, we began by testing for an overall effect of amides using a one-sample t-test to determine whether the removal preference index differed from zero. We then used a GLM with bat species as a predictor variable to determine whether there were differences among the three bat species in their response to the amide treatment. In cases where a significant overall effect of bat species was found, we used a post-hoc Tukey's HSD test to compare the effects among the three species.

To test whether amides affect the proportion of fruit consumed by bats from infructescences once they are removed
(Q2), we again used data pooled by trial and created an index of consumption preference. For each group of bats, we averaged the proportion of fruit consumed from treatment versus control infructescences, using data only from individual infructescences that were removed. We then calculated a preference index for each bat group as the average proportion consumed from treatment infructescences minus the average proportion consumed from control infructescences. A number of trials in which bats did not remove any treatment infructescences were excluded from these analyses. We conducted statistical analyses of this index as above, beginning with a one-sample t-test to examine the overall effects of amides on the proportion of fruit consumed, and proceeding with GLMs to determine how the magnitude of the consumption index was affected by amide treatment, bat species, group size, and all interactions (Table 2). Based on significant overall effects of amide treatment and bat species, we used post-hoc Tukey's HSD tests to compare the effects among the five amide treatments and the three bat species.

To test whether there were dose-dependent effects of $P$. reticulatum ripe fruit amides on fruit consumption (Q3), we used a linear mixed model (LMM) with the total mass of fruit consumed as the response variable, extract concentration as a fixed effect, and bat identity as a random effect. This model was compared using a likelihood ratio test to a null model that included no fixed effects but retained bat identity as a random effect. After finding a significant effect of concentration, we followed this test with a post-hoc Tukey's HSD test to compare the effects among the different concentrations of amides.

All statistical analyses were conducted in $\mathrm{R}$ ver. 2.15.3 using the base functions and the packages lme4 (Bates et al. 2013) for GLMMs and multcomp (Hothorn et al. 2013) for post hoc multiple comparisons.

\section{Data deposition}

Data available from the Dryad Digital Repository: <http:// dx.doi.org/10.5061/dryad.br022> (Whitehead et al. 2015).

\section{Ethics statement}

All experiments described in this study were conducted in accordance with guidelines for the use of animals in research from the American Society of Mammologists and were approved by the Institutional Animal Care and Use Committee (IACUC) at the University of Colorado (protocol no. 1011.04).

\section{Results}

\section{Effects of amides on infructescence removal (Q1)}

Amides had a negative overall effect on the number of infructescences removed by bats (mean removal index $=-1.09, \mathrm{t}=-5.127, \mathrm{DF}=84, \mathrm{p}<0.0001)$, but the effects varied among bat species and depending on the specific compound(s) or amide extract tested. From the starting saturated linear model (removal index $\sim$ amide treatment $\times$ bat species $\times$ group size), model simplification (Table 1) led to a 
Table 1. Candidate models describing the factors affecting the infructescence removal index ${ }^{\mathrm{a}}$.

\begin{tabular}{|c|c|c|c|c|c|}
\hline Model specification ${ }^{b}$ & $\mathrm{AICc}$ & $\triangle \mathrm{AICc}$ & Akaike weight & Single term deletion ${ }^{c}$ & p-valued \\
\hline AmidelD $\times S p \times G S$ & 397.6 & 47.0 & $<0.001$ & & \\
\hline AmideID + Sp + GS + AmidelD $\times S p+$ AmidelD $\times$ GS $+S p \times G S$ & 371.1 & 20.6 & $<0.001$ & AmidelD $\times S p \times G S$ & 0.79 \\
\hline AmidelD + Sp + GS + AmideID $\times S p+S p \times G S$ & 359.0 & 8.5 & 0.009 & AmideID $\times$ GS & 0.68 \\
\hline AmidelD + Sp + GS + AmidelD $\times S p$ & 352.7 & 2.2 & 0.21 & $\mathrm{Sp} \times \mathrm{GS}$ & 0.87 \\
\hline AmidelD + Sp + GS & 353.3 & 2.7 & 0.16 & AmidelD $\times S p$ & 0.003 \\
\hline AmidelD + Sp + AmidelD $\times$ Spe & 350.5 & 0.0 & 0.63 & GS & 0.34 \\
\hline
\end{tabular}

${ }^{a}$ Calculated as the number of treatment infructescences minus the number of control infructescences removed by a group of bats during the trial period.

bFixed effects included the type of amide treatment (AmideID), bat species (Sp), and group size (GS).

cFixed effect term removed from the model in the previous step of model simplification.

dp-value resulting from a likelihood ratio test comparing the specified model to the more complex model that included the deleted term in the single term deletion column. These values may be interpreted as the significance of the effect of the term in the single term deletion column.

eMinimum adequate model.

minimum adequate model that included only the significant effects of amide treatment $(\mathrm{F}=4.00, \mathrm{DF}=4, \mathrm{p}=0.0056)$, bat species $(\mathrm{F}=5.29, \mathrm{DF}=2, \mathrm{p}=0.0072)$, and their twoway interaction $(\mathrm{F}=2.74, \mathrm{DF}=8, \mathrm{p}=0.011)$. Because of the significant interaction between amide treatment and bat species, we further analyzed differences among bat species separately for each amide treatment. For piperine, there was an overall negative effect of treatment on removal (mean removal index $=-1.75, \mathrm{t}=-3.13, \mathrm{DF}=15, \mathrm{p}=0.0069$ ), and the linear model indicated significant differences among the three bat species $(F=5.28, D F=2, p=0.021$; Fig. $2 A$ ). A Tukey HSD post-hoc test for all pairwise comparisons of bat species indicated that $C$. perspicillata $(\mathrm{p}=0.03)$ and $C$. sowelli $(\mathrm{p}=0.05)$ were more deterred by piperine than $C$. castanea. For piplartine, there was no overall negative effect of amide treatment on removal (mean removal index $=-0.23$, $\mathrm{t}=-0.62 \mathrm{DF}=20, \mathrm{p}=0.54)$ and no significant differences among the three bat species $(\mathrm{F}=1.47, \mathrm{DF}=2, \mathrm{p}=0.26$; Fig. 2A). For the piperine and piplartine combination, there was no overall effect of amide treatment on removal (mean removal index $=-0.60, t=-1.5, D F=19, p=0.15)$, but there were significant differences among the three bat species $(F=9.63, D F=2, p=0.0016$; Fig. 2A). A Tukey HSD post-hoc test indicated that the negative effects of the amide combination on $C$. perspicillata $(\mathrm{p}=0.0039)$ and $C$. castanea $(\mathrm{p}=0.0023)$ were stronger relative to the effects on $C$. sowelli, which actually trended towards increased removal of amide-treated infructescences (Fig. 2A, Supplementary material Appendix 5 Table A5a). For P. reticulatum unripe extracts, there was an overall negative effect of treatment on removal (mean removal index $=-2.25, \mathrm{t}=-4.18$, $\mathrm{DF}=11, \mathrm{p}=0.0015)$, and the linear model indicated no significant differences among the three bat species $(F=0.92$, $\mathrm{DF}=2, \mathrm{p}=0.43$; Fig. $2 \mathrm{~A}$ ). For $P$. reticulatum ripe extracts, there was again an overall negative effect of treatment on removal (mean removal index $=-1.31, \mathrm{t}=-3.02, \mathrm{DF}=15$, $\mathrm{p}=0.0087)$, and the linear model indicated no significant differences among the three bat species $(\mathrm{F}=1.41, \mathrm{DF}=2$, $\mathrm{p}=0.28$; Fig. 2A).

\section{Effects of amides on fruit consumption (Q2)}

Amides had a negative overall effect on the proportion of fruit that bats consumed per infructescence (mean consumption index $=-0.19, \mathrm{t}=-5.43, \mathrm{DF}=62, \mathrm{p}<0.0001)$, but the effects varied among bat species and depending on the specific compound(s) or extract tested. From the starting saturated linear model (consumption index $\sim$ amide treatment $\times$ bat species $\times$ group size), model simplification (Table 2 ) led to a minimum adequate model that included only the

Table 2. Candidate models describing the factors affecting the fruit consumption index ${ }^{\text {. }}$

\begin{tabular}{|c|c|c|c|c|c|}
\hline Model specification ${ }^{b}$ & $\mathrm{AICc}$ & $\triangle \mathrm{AICC}$ & Akaike weight & Single term deletion ${ }^{c}$ & p-value \\
\hline AmidelD $\times \mathrm{Sp} \times \mathrm{GS}$ & 78.6 & 65.2 & $<0.001$ & & \\
\hline AmidelD + Sp $+\mathrm{GS}+$ AmidelD $\times \mathrm{Sp}+$ AmidelD $\times \mathrm{GS}+\mathrm{Sp} \times \mathrm{GS}$ & 48.4 & 35 & $<0.001$ & AmidelD $\times \mathrm{Sp} \times \mathrm{GS}$ & 0.34 \\
\hline AmidelD + Sp + GS + AmidelD $\times$ GS $+S p \times G S$ & 23.6 & 10.2 & 0.003 & AmideID $\times S p$ & 0.60 \\
\hline AmideID + Sp + GS + AmideID $\times$ GS & 18.3 & 4.8 & 0.04 & $\mathrm{Sp} \times \mathrm{GS}$ & 0.57 \\
\hline AmideID + Sp + GS & 15.8 & 2.3 & 0.14 & AmidelD $\times$ GS & 0.10 \\
\hline AmidelD + Spe & 13.4 & 0.0 & 0.46 & GS & 0.54 \\
\hline AmidelD & 18.5 & 5.1 & 0.04 & Sp & 0.005 \\
\hline Sp & 14.1 & 0.7 & 0.32 & AmidelD & 0.03 \\
\hline
\end{tabular}

aCalculated as the average proportion of fruit consumed from treatment infructescences minus the average proportion of fruit consumed from control infructescences by a group of bats during the trial period.

bFixed effects included the type of amide treatment (AmideID), bat species (Sp), and group size (GS).

cFixed effect term removed from the model in the previous step of model simplification.

dp-value resulting from a likelihood ratio test comparing the specified model to the more complex model that included the deleted term in the single term deletion column. These values may be interpreted as the significance of the effect of the term in the single term deletion column.

eMinimum adequate model. 

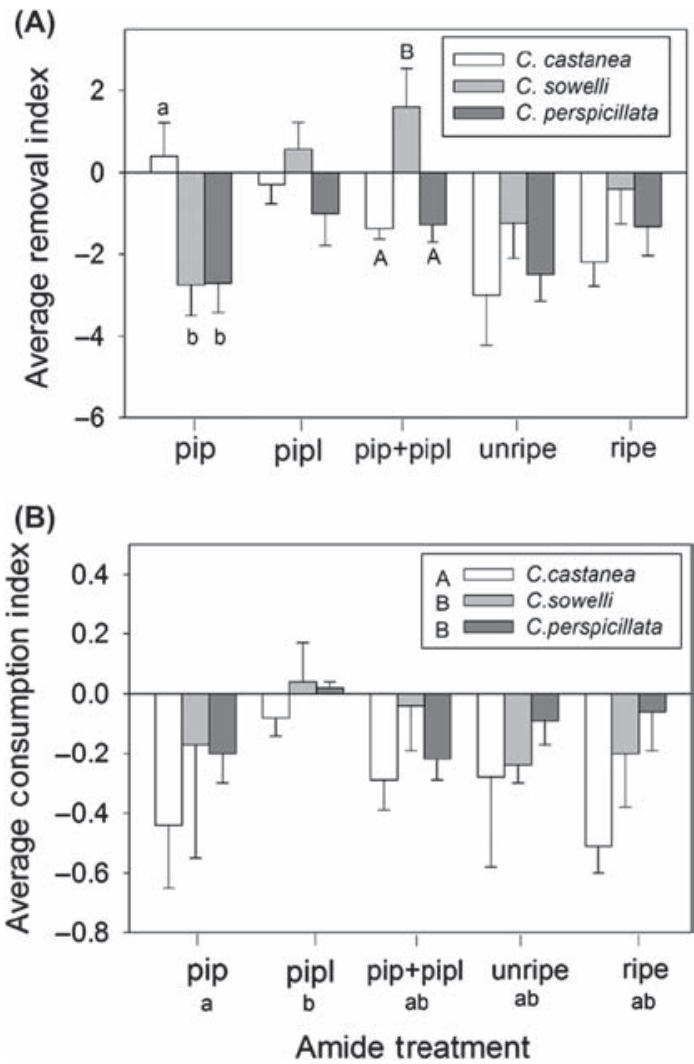

Figure 2. Effects of pure amides and amide-rich extracts on fruit removal (A) and fruit consumption (B) by three species of Carollia bats. Indices were calculated for each trial and the values represent the average $( \pm$ SE) among trials. Negative values of the indices indicate a negative effect of amides on fruit removal or consumption. For fruit removal (A), there was a significant interaction between amide treatment and bat species, and the differences among bat species were analyzed for each amide treatment independently. For piperine and piperine + piplartine treatments only, we found overall effects of bat species and conducted Tukey HSD post-hoc pairwise comparisons among the three species. Letters on bars represent significant differences among bat species. For fruit consumption (B), there was no interaction between amide treatment and bat species. Tukey HSD post-hoc pairwise comparisons were conducted comparing amide treatments without regard to species (lowercase letters) and comparing bat species without regard to amide treatment (uppercase letters).

significant effects of amide treatment $(\mathrm{F}=2.55, \mathrm{DF}=4$, $\mathrm{p}=0.049$; Fig. 2B) and bat species $(\mathrm{F}=4.93, \mathrm{DF}=2$, $\mathrm{p}=0.011$; Fig. 2B). Tukey HSD post-hoc tests for all pairwise comparisons among the individual amides and extracts indicated that the negative effects of piperine and ripe fruit extracts were marginally stronger than those of piplartine $(\mathrm{p}=0.052$ and $\mathrm{p}=0.069$, respectively). Tukey HSD posthoc comparisons among bat species indicated that the negative effects of amides on consumption by $C$. castanea were stronger than the effects on C. perspicillata $(\mathrm{p}=0.014)$ or C. sowelli $(\mathrm{p}=0.050)$. Because of the differences among bat species, we also used one-sample t-tests to determine if there was an overall effect of amides on the proportion of fruit consumed for each species, and found that there was a negative effect of amides on consumption by $C$. castanea (mean consumption index $=-0.32, \mathrm{t}=-5.14, \mathrm{DF}=21$,

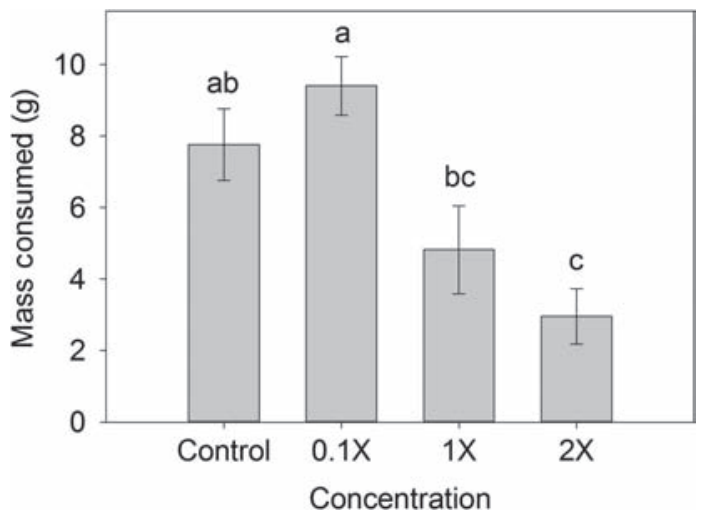

Figure 3. Effects of increasing concentrations of Piper reticulatum ripe fruit extracts on the average ( $\pm \mathrm{SE}$ ) mass of fruit consumed by Carollia perspicillata in no-choice feeding trials. The amount of fruit consumed was measured as the combined lengths from which fruit was consumed from all Piper sancti-felicis infructescences presented during a two hour period and converted to mass. Letters above bars represent significant differences among amide concentration treatments in Tukey HSD post-hoc pairwise comparisons.

$\mathrm{p}<0.0001$ ) and by $C$. perspicillata (mean consumption index $=-0.12, \mathrm{t}=-2.81, \mathrm{DF}=24, \mathrm{p}=0.0098)$, but not by $C$. sowelli (mean consumption index $=-0.12, \mathrm{t}=-1.61$, $\mathrm{DF}=15, \mathrm{p}=0.13)$.

\section{Dose-dependent effects of ripe fruit amides on fruit consumption (Q3)}

Increasing concentrations of amide-rich extracts significantly reduced the amount of fruit consumed by individual bats from fruit buffets in no-choice feeding experiments $\left(\chi^{2}=20.82, \mathrm{DF}=3, \mathrm{p}=0.00011\right.$; Fig. 3). Pairwise comparisons among controls and the three concentrations tested showed that significantly less fruit was consumed from infructescences supplemented at $2 \mathrm{X}$ than from controls $(p=0.0027)$, but controls were not different from the $0.1 X$ $(p=0.63)$ or $1 X(p=0.14)$ treatments. The most fruit was consumed from the infructescences supplemented at $0.1 \mathrm{X}$; this treatment was different from the 1X $(p=0.0048)$ and $2 \mathrm{X}(\mathrm{p}<0.001)$ treatments.

\section{Discussion}

Secondary metabolites can increase plant fitness by defending plant tissues against herbivores and pathogens (Iason et al. 2012), but they also have important consequences for interactions with mutualists, such as pollinators and seed dispersers (Adler 2000, Levey et al. 2007). This study examined how secondary metabolites in fleshy fruits can affect the foraging and feeding behavior of seed-dispersing bats. Our results showed that amides, an important class of plant defensive compounds (Dyer et al. 2004), have generally negative effects on bat preferences for Piper fruit, both in terms of the removal of infructescences and the proportion of fruit that bats consume from an infructescence once they begin to feed (average removal and consumption indices significantly less than zero, $p<0.0001$; Fig. 2). Furthermore, these effects 
appear to be dose-dependent within the range of amides present in natural Piper infructescences (Fig. 3). Thus, in the case of amides in Piper, our data support the hypothesis of a tradeoff between seed dispersal and fruit defense (Herrera 1982, Cipollini and Levey 1997a) over the hypothesis of microbe-pest specificity, which suggests that defensive compounds can be specific to pests with limited effects on mutualists (Cipollini and Levey 1997a). However, we also found that the effects of amides varied considerably depending on the bat species and specific compound(s) involved (Fig. 2). These results suggest that the strength of the tradeoff may depend on a variety of environmental factors and provide general support for the idea that fruit-frugivore interactions can be highly context dependent (Perea et al. 2013).

Our results for the effects of amides on removal clearly show that bats can detect amides prior to removal and have reduced preferences for amide-supplemented Piper fruits (Fig. 2A). This finding was somewhat contrary to our expectations, because amides have very low volatility and are not likely to be a major component of Piper fruit odor. It is possible that removal choices were, at least in some cases, informed by a combination of taste and odor, because bats did often make a number of exploratory flights or removal attempts at a single infructescence before they finally removed it in flight (behavior described in detail in Thies et al. 1998). In addition, bats were able to make multiple choices over the course of a trial and may have learned to avoid amide-supplemented fruits; however, the pattern of reduced removal of amidesupplemented infructescences held even for the first removal event of the trials and was not significantly explained by individual learning (Supplementary material Appendix 4). It is also noteworthy that the effects of amides on removal were highly variable for different bat species and amide treatments (Fig. 2A). For example, in our trials with C. sowelli and the piperine/piplartine combination, there was an unexpected trend towards increased removal of amide-supplemented infructescences (Fig. 2A; Supplementary material Appendix 5 Table A5a). We further explored these results by testing for potential non-additive effects of piperine and piplartine when presented in mixture and found marginally significant evidence for compound interactions (Supplementary material Appendix 6). These same two compounds have also been shown to interact synergistically or antagonistically in the defense of Piper fruits against insect and microbial pests (Whitehead and Bowers 2014), and, furthermore, could interact with other chemical cues used by bats in unknown ways. Thus, the potential for interactions among compounds to influence the fruit removal preferences of bats under field conditions warrants further investigation. It is possible that bat foraging decisions depend not just on total amide concentrations, but on the particular combinations of compounds that are present. While our results suggest that amides generally have a negative effect on removal preferences, it is possible that certain compound combinations (such as piperine + piplartine, Fig. 2A) could be preferred by bats, especially if they are used by bats as foraging cues that allow them to recognize particular Piper species (Cipollini and Levey 1997a).

Our results also show that amides can have a negative effect on the proportion of fruit that bats consume from an infructescence once it is removed. This effect was strongest for C. castanea, intermediate for C. perspicillata, and there was no overall negative effect of amides for $C$. sowelli (Fig. 2B). Whether or not bats consume an entire infructescence once they begin feeding might be in part explained in the framework of optimal foraging theory, which predicts that animal foraging behavior will reflect selection to maximize net energy gain (Pyke 1984). Once bats have expended the energy to locate and remove a ripe infructescence, in most cases the energetic gain would be maximized by consuming it in its entirety. However, if the digestion or detoxification of secondary metabolites also represents a substantial energy expenditure, then bats may maximize energetic gain by keeping the amount of secondary metabolites ingested below a certain threshold. This may help explain why $C$. perspicillata and $C$. sowelli, which overall have a more varied diet than C. castanea (Fleming 1991), and therefore would likely consume lower total amounts of amides in a single evening, may be less constrained by the amount of secondary metabolites consumed in any one infructescence. There is some evidence suggesting that bats that consume higher proportions of fruit require some means of secondary metabolite detoxification - in a previous study with $C$. perspicillata in the Ecuadorian Amazon, individuals with a higher percentage of fruit relative to insects in their diet spent more time visiting mineral licks, which are thought to buffer the effects of secondary metabolites and aid in detoxification (Voigt et al. 2008).

Various factors may contribute to differences among Carollia species in their responses to different amide treatments. One possibility is that there is some differential specialization within the genus, where certain Carollia species consume relatively higher amounts of certain species of Piper, and therefore may have evolved an ability to tolerate or detoxify the specific compounds that are more abundant in their diet. Another factor contributing to the variation in response among Carollia species may be variation in habitat use and the availability of different Piper species in their typical foraging range. Our results suggest that $C$. sowelli is the least deterred by amides, both in terms of fruit removal and fruit consumption (Fig. 2). This species has been less well-studied than its congeneric counterparts, but one study showed that $C$. sowelli is the most sedentary (i.e. has the smallest foraging range) of the three species co-occurring at La Selva (Fleming 1991). If this smaller range means there are fewer food options available to individuals at any given point in time, it could result in $C$. sowelli being the least 'choosy' about available food items.

For one species, $C$. perspicillata, the negative effects of amides on bat preferences were further supported by a set of experiments conducted to examine how varying concentrations of amides affect fruit consumption by bats in a no-choice context. In cases where fruiting Piper shrubs are separated spatially, this no-choice scenario may provide a better approximation of the foraging decisions bats make in field conditions. We found that $P$. sancti-felicis fruits supplemented with the average amount of amides found in P. reticulatum ( $1 \mathrm{X}$ treatment) had no deterrent effect relative to controls, but those fruit supplemented with twice the average amount ( $2 \mathrm{X}$ treatment) were deterrent. Natural concentrations of amides in ripe fruits of $P$. reticulatum can vary over 10-fold (from $0.16-2 \%$ dry weight; Whitehead et al. 2013) and the $2 X$ treatment is still well within the range of 
natural variation. Thus, a deterrent effect of the $2 \mathrm{X}$ amide treatment relative to controls or low concentration ( $0.1 \mathrm{X})$ treatments suggests that the natural variation in amide concentration among Piper plants is sufficient to result in variation in the strength of any potential tradeoff between seed dispersal and fruit defense.

Although our results provide support for the hypothesis of trade-offs between seed dispersal and fruit defense, the ultimate fitness consequences of this tradeoff for plants will depend on how reduced bat preferences for fruits translate to altered seed germination and seedling establishment. Piper infructescences with higher concentrations of amides may be only partially consumed, but it is unclear whether seeds that are dropped below feeding roosts inside partially intact infructescences are able to survive and germinate with similar probability compared to seeds that are consumed and defecated. Although the germination probability is similar for cleaned Piper seeds that are collected from bat feces versus ripe fruits (Palmeirim et al. 1989); many studies have shown that cleaned seeds have a much higher germination probability than seeds inside intact fruits (Traveset et al. 2007). This could be due to chemical inhibition of seed germination by pulp metabolites (Traveset et al. 2007) or due to rapid fungal attack and decomposition of unconsumed ripe fruit (Obando and Whitehead unpubl.). However, at least in some cases, seed germination is likely from partially consumed fruits (Fedriani et al. 2012), and the probability of germination in partially eaten Piper infructescences is unknown. Other interacting factors may also influence the relationship between fruit amides and germination success. We have observed that intact or partially consumed Piper infructescences on the forest floor are sometimes rapidly removed piecemeal by foraging ants, and the effects of amides on ant dispersal are unknown. In addition, certain amides have been shown to speed the passage rates of seeds through bat guts, which can have important implications for both dispersal distance and seed viability (Baldwin and Whitehead 2015b). Clearly, understanding the true fitness consequences of secondary metabolites in ripe fruits is complex and will require in depth study of seed survival and germination in a variety of scenarios.

There are two important caveats to our results. First, bat responses were measured in cages with a plentiful supply of infructescences, and responses in this environment may differ from those in natural habitats. If Piper infructescences represent a limited resource, bats may be less choosy and accept higher concentrations of secondary metabolites. Second, we measured the effects of amides by adding compounds to infructescences of Piper sancti-felicis, a species that does not naturally contain amides. This approach allowed us to observe the natural behaviors of Carollia bats feeding on Piper infructescences while avoiding the confounding effects of any underlying variation in amide content. However, it did create a novel combination of secondary metabolites that could interact in unknown ways and that bats have not experienced in natural habitats. One alternative explanation for our results is that bats avoided amidesupplemented infructescences simply because they were novel. Although this cannot be ruled out as a partial explanation for our results, we do not believe it can fully account for the deterrent effects of amides for two main reasons. First, while all amide treatments were novel with respect to their occurrence in $P$. sancti-felicis, not all treatments were avoided (e.g. piplartine and low concentrations of amides in our nochoice tests). Also, we conducted preliminary trials with $C$. perspicillata where amides were added to a completely novel food (banana agar diet mix), and still found that amides were deterrent relative to the unsupplemented controls (Whitehead unpubl.).

This study has provided evidence that amides can negatively affect the foraging and feeding preferences of Carollia bats. Combined with our previous work showing that the same amides can deter insect seed predators and reduce the growth rates of fungi (Whitehead and Bowers 2014), these results suggest that amides in Piper fruits likely represent a tradeoff between defense against antagonists and attraction of mutualist seed dispersers. However, understanding the ultimate consequences of fruit amides for plant reproductive fitness will require additional well-replicated experiments in field conditions to examine how amides affect seed germination and survival in natural habitats. Furthermore, because our results show that even closely-related species (three bats in the same genus) can be affected in different ways by specific compounds or combinations of compounds in fruits, future work should focus on understanding whether there is subgeneric specialization in seed dispersal interactions within the genus Piper. The divergent selective pressures imposed by different Carollia species or by different disperser groups (e.g. bats versus birds) may be one factor contributing to the chemical evolution and diversity of the genus Piper (Parmar et al. 1997). Although secondary metabolites have primarily been examined as defensive traits in leaves, the potential for fruit secondary metabolites to affect multiple mutualistic and antagonistic interactions in which plants are simultaneously involved may be an important and underappreciated force in determining the outcome of ecological interactions and the evolution of plant chemical diversity.

Acknowledgements - Statement of authorship: SRW and MDB designed experiments, SRW and MFO developed methodology and performed experiments, SRW conducted data analysis, SRW wrote the manuscript and all authors contributed substantially to revisions. The authors declare no conflict of interest. This research was supported by National Science Foundation (NSF) grant DEB 1210884 to SRW and MDB, a National Geographic Waitt Grant to SRW, an Organization for Tropical Studies Research Fellowship to SRW, and the Department of Ecology and Evolutionary Biology at the University of Colorado. We are grateful to Daniela Azofeifa and Eric Ballestero for assistance with field work. The Organization for Tropical Studies, especially Bernal Matarrita and Danilo Brenes, provided logistical support at La Selva Biological Station, and Javier Guevara from the Ministerio del Ambiente y Energía in Costa Rica assisted with permits (permit no. 075-2012-SINAC). Helpful comments on the manuscript were provided by Lee A. Dyer, William Bowman, Michael Breed and Alexander Cruz.

\section{References}

Adler, L. 2000. The ecological significance of toxic nectar. - Oikos 91: 409-420.

Baldwin, J. and Whitehead, S. R. 2015a. Data from: Fruit secondary compounds mediate the retention time of seeds in the 
guts of Neotropical fruit bats. Dryad Digital Repository $<$ http://dx.doi.org/10.5061/dryad.51q1p $>$.

Baldwin, J. W. and Whitehead, S. R. 2015b. Fruit secondary compounds mediate the retention time of seeds in the guts of Neotropical fruit bats. - Oecologia 177: 453-466.

Bates, D. et al. 2013. lme4: linear mixed-effects models using Eigen and S4. Ver. 1.0-4.

Bezerra, D. P. et al. 2013. Overview of the therapeutic potential of piplartine (piperlongumine). - Eur. J. Pharm. Sci. 48: 453-463.

Cazetta, E. et al. 2008. Does attraction to frugivores or defense against pathogens shape fruit pulp composition? - Oecologia 155: 277-286.

Cipollini, M. L. 2000. Secondary metabolites of vertebratedispersed fruits: evidence for adaptive functions. - Rev. Chil. Hist. Nat. 73: 421-440.

Cipollini, M. L. and Levey, D. J. 1997a. Secondary metabolites of fleshy vertebrate-dispersed fruits: adaptive hypotheses and implications for seed dispersal. - Am. Nat. 150: 346-372.

Cipollini, M. L. and Levey, D. J. 1997b. Why are some fruits toxic? Glycoalkaloids in Solanum and fruit choice by vertebrates. - Ecology 78: 782-798.

Crawley, M. J. 2007. The R book. - Wiley.

de Oliveira Chaves, M. C. et al. 2003. Amides from Piper tuberculatum fruits. - Fitoterapia 74: 181-183.

Dyer, L. A. et al. 2004. Isolation, synthesis, and evolutionary ecology of Piper amides. - In: Dyer Palmer, A. D. N. (ed.), Piper: a model genus for studies in phytochemistry, ecology and evolution. Kluwer, pp. 117-139.

Fedriani, J. M. and Boulay, R. 2006. Foraging by fearful frugivores: combined effect of fruit ripening and predation risk. - Funct. Ecol. 20: 1070-1079.

Fedriani, J. M. et al. 2012. Thieves or mutualists? Pulp feeders enhance endozoochore local recruitment. - Ecology 93: $575-587$.

Fleming, T. 1991. The relationship between body size, diet, and habitat use in frugivorous bats, genus Carollia (Phyllostomidae). - J. Mammal. 72: 493-501.

Fleming, T. H. 2004. Dispersal ecology of neotropical Piper shrubs and treelets. - In: Dyer Palmer, A. D. N. (ed.), Piper: a model genus for studies of phytochemistry, ecology and evolution. Kluwer, pp. $58-77$.

Greig, N. 1993. Regeneration mode in neotropical Piper - habitat and species comparisons. - Ecology 74: 2125-2135.

Haak, D. C. et al. 2012. Why are not all chilies hot? A tradeoff limits pungency. - Proc. R. Soc. B 279: 2012-2017.

Herrera, C. M. 1982. Defense of ripe fruit from pests - its significance in relation to plant-disperser interactions. - Am. Nat. 120: 218-241.

Hodgkison, R. et al. 2007. Chemical ecology of fruit bat foraging behavior in relation to the fruit odors of two species of paleotropical bat-dispersed figs (Ficus hispida and Ficus scortechinii). - J. Chem. Ecol. 33: 2097-2110.

Hothorn, T. et al. 2013. multcomp: simultaneous inference in general parametric models. Ver. 1.3-0.

Iason, G. R. et al. 2012. The ecology of plant secondary metabolites: from genes to global processes. - Cambridge Univ. Press.

Izhaki, I. 2002. Emodin - a secondary metabolite with multiple ecological functions in higher plants. - New Phytol. 155: 205-217.

Karasov, W. H. et al. 2012. Capacity for absorption of watersoluble secondary metabolites greater in birds than in rodents. - PLoS ONE 7: e32417.

Kato, M. J. and Furlan, M. 2007. Chemistry and evolution of the Piperaceae. - Pure Appl. Chem. 79: 529-538.

Kunz, T. H. and Parsons, S. 2009. Ecological and behavioral methods for the study of bats. - The Johns Hopkins Univ. Press.
Larrinaga, A. R. 2010. A univariate analysis of variance design for multiple-choice feeding-preference experiments: a hypothetical example with fruit-eating birds. - Acta Oecol. 36: $141-148$.

Laska, M. 1990. Olfactory sensitivity to food odor components in the short-tailed fruit bat, Carollia perspicillata (Phyllostomidae, Chiroptera). - J. Comp. Phsiol. A 166: 395-399.

Leitáo da-Cunha, E. V and de Oliveira Chaves, M. C. 2001. Two amides from Piper tuberculatum fruits. - Fitoterapia 72: 197-199.

Levey, D. J. et al. 2007. Evolutionary ecology of secondary compounds in ripe fruit: case studies with capsaicin and emodin. - In: Dennis, A. J. et al. (eds), Seed dispersal: theory and its application in a changing world. CABI Publ., pp. 37-58.

Lomáscolo, S. et al. 2010. Dispersers shape fruit diversity in Ficus (Moraceae). - Proc. Natl Acad. Sci. USA 107: 14668-14672.

Mason, J. et al. 1991. Taxon-specific differences in responsiveness to capsaicin and several analogues: correlates between chemical structure and behavioral aversiveness. - J. Chem. Ecol. 17: 2539-2551.

Muscarella, R. and Fleming, T. H. 2007. The role of frugivorous bats in tropical forest succession. - Biol. Rev. 82: 573-590.

OTS 2012. Organization for Tropical Studies Database: La Flora Digital de La Selva. - < http://sura.ots.ac.cr/local/florula3/en/ index.htm $>$. Accessed on 12/10/12.

Palmeirim, J. M. et al. 1989. Trophic structure of a neotropical frugivore community - is there competition between birds and bats? - Oecologia 79: 403-411.

Parmar, V. S. et al. 1997. Phytochemistry of the genus Piper. - Phytochemistry 46: 597-673.

Perea, R. et al. 2013. Context-dependent fruit-frugivore interactions: partner identities and spatio-temporal variations. - Oikos 122: 943-951.

Pyke, G. H. 1984. Optimal foraging theory: a critical review. - Annu. Rev. Ecol. Syst. 15: 523-575.

Rajopadhye, A. A. et al. 2011. HPTLC method for analysis of piperine in fruits of Piper species. - J. Planar Chromatogr. TLC 24: 57-59.

Roa, R. 1992. Design and analysis of multiple-choice feedingpreference experiments. - Oecologia 89: 509-515.

Schaefer, H. M. et al. 2003. Testing the defence tradeoff hypothesis: how contents of nutrients and secondary compounds affect fruit removal. - Oikos 102: 318-328.

Struempf, H. et al. 1999. The cyanogenic glycoside amygdalin does not deter consumption of ripe fruit by cedar waxwings. - Auk 116: 749-758.

Tewksbury, J. J. and Nabhan, G. P. 2001. Directed deterrence by capsaicin in chillies. - Nature 412: 403-404.

Tewksbury, J. J. et al. 2008. Evolutionary ecology of pungency in wild chilies. - Proc. Natl Acad. Sci. USA 105: 11808-11811.

Thies, W. and Kalko, E. K. V 2004. Phenology of Neotropical pepper plants (Piperaceae) and their association with their main dispersers, two short-tailed fruit bats, Carollia perspicillata and C. castanea (Phyllostomidae). - Oikos 104: 362-376.

Thies, W. et al. 1998. The roles of echolocation and olfaction in two Neotropical fruit-eating bats, Carollia perspicillata and C. castanea, feeding on Piper. - Behav. Ecol. Sociobiol. 42: 397-409.

Traveset, A. et al. 2007. A review on the role of endozoochory on seed germination. - In: Dennis, A. et al. (eds), Seed dispersal: theory and its application in a changing world. CABI Publ., pp. $78-103$.

van der Pijl, L. 1982. Principles of dispersal in higher plants. - Springer. 
Voigt, C. C. et al. 2008. Nutrition or detoxification: why bats visit mineral licks of the Amazonian rainforest. - PLoS ONE 3: e2011.

Whitehead, S. R. 2013. Ecological costs and benefits of secondary metabolites in animal-dispersed fruits. - PhD thesis, Univ. of Colorado at Boulder.

Whitehead, S. R. and Poveda, K. 2011. Herbivore-induced changes in fruit-frugivore interactions. - J. Ecol. 99: 964-969.

Whitehead, S. and Bowers, M. 2013. Evidence for the adaptive significance of secondary compounds in vertebrate-dispersed fruits. - Am. Nat. 182: 563-577.

Supplementary material (available online as Appendix oik.02210 at $<$ www.oikosjournal.org/readers/appendix $>$ ). Appendix 1-6.
Whitehead, S. R. and Bowers, M. D. 2014. Chemical ecology of fruit defence: synergistic and antagonistic interactions among amides from Piper. - Funct. Ecol. 28: 1094-1106.

Whitehead, S. R. et al. 2013. Patterns of secondary metabolite allocation to fruits and seeds in Piper reticulatum. - J. Chem. Ecol. 39: 1373-1384.

Whitehead, S. R. et al. 2015. Data from: Chemical tradeoffs in seed dispersal: defensive metabolites in fruits deter consumption by mutualist bats. Dryad Digital Repository $<$ http://dx.doi.org/10.5061/dryad.br022>. 